\title{
Prompt Mechanisms for Online Auctions
}

\author{
Richard Cole Shahar Dobzinski Lisa Fleischer
}

November 12, 2007

\begin{abstract}
We study the following online problem: at each time unit, one of $m$ identical items is offered for sale. Bidders arrive and depart dynamically, and each bidder is interested in winning one item between his arrival and departure. Our goal is to design truthful mechanisms that maximize the welfare, the sum of the utilities of winning bidders.

We first consider this problem under the assumption that the private information for each bidder is his value for getting an item. In this model constant-competitive mechanisms are known, but we observe that these mechanisms suffer from the following disadvantage: a bidder might learn his payment only when he departs. We argue that these mechanism are essentially unusable, because they impose several seemingly undesirable requirements on any implementation of the mechanisms.

To crystalize these issues, we define the notions of prompt and tardy mechanisms. We present two prompt truthful mechanisms - one deterministic and the other randomized, that guarantee a constant competitive ratio. We also prove that our deterministic mechanism is optimal for this setting.

We then study a model in which both the value and the departure time are private information. While in the deterministic setting only a trivial competitive ratio can be guaranteed, we use randomization to obtain a prompt truthful $\Theta\left(\frac{1}{\log m}\right)$-competitive mechanism. Finally, we show that no truthful randomized mechanism can achieve a ratio better than $\frac{1}{2}$ in this model.
\end{abstract}

\section{Introduction}

\section{$1.1 \quad$ Background}

The field of algorithmic mechanism design attempts to handle the strategic behavior of selfish agents in a computationally feasible way. To date, most work in this field has sought to design truthful mechanisms for static settings, such as auctions. In reality, however, the setting of many problems is online, meaning that the mechanism has no prior information regarding the identity of the participating players, or that the goods that are for sale are unknown in advance. Examples include sponsored search auctions [12], single-good auctions [10], and even pricing WiFi at Starbucks [5].

This paper considers the following online auction problem: at each time unit exactly one of $m$ identical items is offered for sale. The item at time $t$ is called item $t$. There are $n$ bidders, where bidder $i$ arrives at time $a_{i}$ and departs at time $d_{i}$, both unknown before bidder $i$ 's arrival. The interval $\left[a_{i}, d_{i}\right]$ will be called bidder $i$ 's time window, and the set of items offered in $i$ 's time window will be denoted by $W_{i}$. Each bidder is interested in winning at most one of the items within $W_{i}$. Let $v_{i}$ denote the value to the $i$ th bidder of getting an item in $W_{i}$. Our goal is to maximize the social welfare: the sum of the values of the bidders that get some item within their time window. As usual in online algorithms, are goal is to optimize the competitive ratio: the worst-case ratio between the welfare achieved by the algorithm and the optimal welfare.

This problem is equivalent to scheduling unit-length jobs on a single machine. In the non-strategic setting, this problem and its variants have been widely studied (e.g., $[1,8,3])$. The best deterministic algorithm to date guarantees a competitive ratio of $\approx 0.547[4,11]$, while it is known that no 
deterministic algorithm can obtain a ratio better than $\frac{2}{\sqrt{5}+1} \approx 0.618$ [2]. In the randomized setting, a competitive ratio of $1-\frac{1}{e}$ is achieved by [1], and no algorithm can achieve a ratio better than 0.8 [2].

This scheduling problem provides an excellent example of the extra barriers we face when designing online mechanisms. The only general technique known for designing truthful mechanisms is the VCG payment scheme. In the offline setting we can obtain an optimal solution in polynomial time (with bipartite matching), and then we can apply VCG. In the online setting, however, it is impossible to find an optimal solution, and thus we cannot use VCG. Yet, truthful competitive mechanisms do exist. The competitive ratio of these mechanisms depends on the specific private-information model each mechanism was designed for. This paper considers two different natural models:

- The Value-Only model: Here, the private information of bidder $i$ consists of only his value $v_{i}$, and the arrival time and the departure time are known to all (but both are unknown prior to the arrival of bidder $i$ ).

- The Generalized Model: The private information of bidder $i$ consists of two numbers: his value $v_{i}$ and his departure time $d_{i}$. The arrival time is public information (but unknown prior to the arrival of bidder $i$ ).

\subsection{The Value-Only Model: Is Monotonicity Enough?}

The only private information of a bidder in the value-only model is his value, and thus this model falls under the category of single-parameter environments - environments in which the private information of each bidder consists of only one number. Fortunately, designing truthful mechanisms for singleparameter environments is quite well understood: an algorithm is truthful if and only if it is monotone. That is, a winning bidder that raises his bid remains a winner.

Using the above characterization, it is possible to prove that the greedy algorithm is monotone [7] (see Section 2.4 for a description). Together with the result of [8], this gives a truthful mechanism that is $\frac{1}{2}$ competitive.

However, a closer look at this mechanism may make one wonder if it is indeed applicable. The notions of prompt and tardy mechanisms we define next highlight the issue.

Definition 1.1 We say that a mechanism for the scheduling problem is prompt if a bidder that wins an item always learns his payment immediately after winning the item. A mechanism is tardy if it is not prompt.

The tardiness in the above mechanism [7,8] is substantial: there are inputs for which a bidder learns his payment only when he departs. Tardy mechanisms seem very unintuitive for the bidders, and in addition they suffer from the following disadvantages:

- Uncertainty: A winning bidder does not know the cost of the item that he won, and thus does not know how much money he still has available. E.g., suppose the mechanism is used in a Las Vegas ticket office for selling tickets to a daily show. A tourist that wins a ticket is uncertain of the price of this privilege, and thus might not be able to determine how much money he has left to spend during his Las Vegas vacation.

- Debt Collection: A winning bidder might pay the mechanism long after he won the item. A bidder that is not honest may try to avoid this payment. Thus, the auctioneer must have some way of collecting the payment of a winning bidder.

- Trusted Auctioneer: A winning bidder essentially provides the auctioneer with a "blank check" in exchange for the item. Consequently, all bidders must trust the honesty of the auctioneer. 
Even if the bidders trust the auctioneer, they may still want to verify the exact calculation of the payment, to avoid over-payments that make winning the item less profitable, or even unprofitable. In order to verify this calculation, the bids of all bidders have to be revealed, leading to an undesirable loss of privacy.

Notice that all of these problems are due to the online nature of the setting, and do not arise in the offline setting. To overcome these problems, we present prompt mechanisms for the scheduling problem. Prompt mechanisms are very intuitive to the bidders as they (implicitly) correspond to takeit-or-leave-it offers: a winning bidder is offered a price for one item exactly once before getting the item, and may reject the offer if it is not beneficial for him. We improve upon the greedy algorithm of $[7,8]$ by showing a different mechanism that achieves the same competitive ratio, but is also prompt.

Theorem: There exists a $\frac{1}{2}$-competitive prompt and truthful mechanism for the scheduling problem in the value-only model.

We show that this is the best possible by proving that no prompt deterministic mechanism can guarantee a competitive ratio better than $\frac{1}{2}$.

We also present a randomized mechanism that guarantees a constant competitive ratio. The achieved competitive ratio of the latter algorithm is worse than the competitive ratio of the deterministic algorithm. Yet, the core of the proof studies a balls-and-bins problem that might be of independent interest.

\subsection{The Generalized Model}

While truthful mechanisms for single-parameter settings are well characterized and thus relatively easy to construct, truthful mechanisms for multi-parameter settings, like the generalized model, are much harder to design. The online setting considered in this paper only makes the design of truthful mechanisms a more challenging task.

The scheduling problem in the generalized model illustrates this challenge. Lavi and Nisan [9] introduced the scheduling problem to the mechanism design community. They showed that no truthful deterministic mechanism for this multi-parameter problem can provide more than a trivial competitive ratio. As a result, Lavi and Nisan suggested looking for a weaker solution concept, set-nash, and provided mechanisms with a constant competitive ratio under this notion. We stress that the set-nash solution concept is much weaker than the dominant-strategy truthfulness considered in this paper.

By contrast with [9], instead of relaxing the solution concept, we use the well-known idea that randomization can help in mechanism-design settings [14]. We provide randomized upper and lower bounds in the generalized model for the scheduling problem.

Theorem: There exists a prompt truthful randomized $\Theta\left(\frac{1}{\log m}\right)$-competitive mechanism for the scheduling problem in the generalized model.

The main idea of the mechanism is to extend the randomized mechanism for the value-only model to the generalized model. Specifically, we use the random-sampling method introduced in [6] in order to be able to "guess" the departure time of each bidder. This mechanism is also a prompt mechanism. We notice that it is quite easy to obtain mechanisms with a competitive guarantee of the logarithm of the ratio between the highest and lowest valuations. However, since this ratio might be exponential in the number of items or bidders, this guarantee is quite weak. By contrast, the competitive ratio our mechanism achieves is independent of the ratio between the highest and lowest valuations, and the mechanism is not required to know the highest and lowest valuations in advance.

Theorem: No truthful randomized mechanism for the scheduling problem in the generalized model can obtain a competitive ratio better than $\frac{1}{2}$. 
The proof of this bound is quite complicated. We start by defining a family of recursively-defined distributions on the input, and then show that no deterministic mechanism can obtain a competitive ratio better than $\frac{1}{2}$ on this family of distributions. We then use Yao's principle to derive the theorem.

The main open question left in the generalized model is to determine whether there is a truthful mechanism with a constant competitive ratio.

\section{Paper Organization}

In Section 2 we describe prompt mechanisms for the value-only case, and prove a that no deterministic tardy algorithms can achieve a ratio better than $\frac{1}{2}$. Lower and upper bounds for the generalized case are proved in Section 3.

\section{Prompt Mechanisms and the Value-Only Model}

\subsection{A Deterministic Prompt $\frac{1}{2}$-Competitive Mechanism}

The mechanism maintains a candidate bidder $c_{j}$ for each item $j$. To keep the presentation simple and without loss of generality, we assume an initialization of the mechanism in which each item $j$ receives a candidate bidder $c_{j}$ with a value of 0 for winning an item (i.e., $v_{c_{j}}=0$ ).

The mechanism runs as follows: at each time $t$ we look at all the bidders that arrived at time $t$. We consider these bidders one by one in some arbitrary order (independent of the bids): for each bidder $i$ we look at the set of all candidates in $i$ 's time window, and let $c_{j}$ be the candidate bidder with the smallest bid (if there are several such candidates select one arbitrarily). Formally, $c_{j} \in \arg \min _{k \in W_{i}} c_{k}$. We say that $i$ competes on item $j$. Now, if $v_{c_{j}}<v_{i}$, we make $i$ the candidate bidder for item $j$. After all the bidders that arrived in time $t$ have been processed, we allocate item $t$ to the candidate bidder $c_{t}$.

The next theorem proves that this algorithm is monotone, i.e., a bidder that raises his bid is still guaranteed to win. This is also a necessary and sufficient condition for truthfulness. We are still left with the issue of finding the payments themselves. First, observe that the payment of each winning bidder must equal his critical value: the minimum value he can declare and still win. Notice that this value is indeed well defined if the algorithm is monotone. For each bidder $i$ this value can be found by using a binary search on the values in $i$ 's domain. Clearly, this procedure takes a polynomial time. See, e.g., [13] for a more thorough discussion. By the discussion above, it is clear that a mechanism is prompt if and only if $i$ 's critical value can be found by the time a $i$ wins an item. In this case, the payment can also be calculated in polynomial time.

Theorem 2.1 The above mechanism is prompt and truthful. Its competitive ratio is $\frac{1}{2}$.

Proof: To show that the mechanism is truthful we have to show that it is monotone: that is, a winning bidder $i$ still wins an item by raising his value $v_{i}$ to $v_{i}^{\prime}$. First, observe that fixing the declarations of the other bidders, $i$ competes on item $j$ regardless of his value. We now compare two runs of the mechanism, with $i$ declaring $v_{i}$ and with $i$ declaring $v_{i}^{\prime}$, and show that at each time the candidate for any item $j^{\prime}$ is the same in both runs. In particular, it follows that the set of winners stays the same, and thus the mechanism is monotone.

Look at the next bidder $e$ that arrives after $i$. For a contradiction, suppose that the candidate for some item changes after bidder $e$ arrives. It follows that $i$ declaring $v_{i}^{\prime}$ causes $e$ to compete on an item different than the one that $e$ competes on when $i$ declares $v_{i}$. This is possible only if $e$ is competing on $j$ if $i$ declares $v_{i}$, but if $i$ declares $v_{i}^{\prime}$, e competes on $h \neq j$. It follows that if $i$ declares $v_{i}^{\prime}$ both $i$ and $e$ compete on $j$, and that $i$ wins $j$. Thus, $v_{i} \geq v_{e}$. When $i$ raises his bid $e$ competes on $h$. Let $c_{h}$ be the candidate for $h$ at the time that $e$ arrives. We have that $v_{i}^{\prime}>v_{c_{h}}>v_{i}$, and thus $v_{e}<v_{c_{h}}$ so $e$ 
does not become a candidate on $h$, and the set of candidates stays the same. To finish the proof for the monotonicity of the mechanism, we look at the rest of the bidders one by one, and repeat the same arguments.

As for the promptness of the mechanism, observe that the identity of the item that $i$ competes on is determined only by the information provided by bidders that had already arrived by the time of $i$ 's arrival. The winner of any item $j$ is of course completely determined by the information provided by bidders that arrived by time $j$. Thus, we can calculate the payment of a winning bidder immediately after he wins an item.

We now analyze the competitive ratio of the mechanism. Let $O P T=\left(o_{1}, \ldots, o_{m}\right)$ be the optimal solution, and $A L G=\left(p_{1}, \ldots, p_{m}\right)$ be the solution constructed by the mechanism. That is, $o_{j}$ is the bidder that wins item $j$ in $O P T$ and $p_{j}$ is the bidder that wins item $j$ in $A L G$. We will match each bidder $i$ that wins an item in OPT to exactly one bidder $l$ that wins an item in ALG. Furthermore, we will make sure that $v_{i} \leq v_{l}$, and that each bidder in ALG is associated with at most two bidders in OPT. This is enough to prove a competitive ratio of $\frac{1}{2}$.

The bidders are matched as follows: for each item $j$, let $o_{j_{1}}, \cdots, o_{j_{k_{j}}}$ be the bidders (ordered by their arrival time) that won an item in the optimal solution and are competing on $j$. Now match each $o_{j_{r}}$ to $p_{j_{r+1}}$ for $r<k_{j}$. Match $o_{j_{k_{j}}}$ to $p_{j}$, the bidder that wins $j$ in ALG (it is possible that $p_{j}=o_{j_{k_{j}}}$ ).

Observe that bidder $p_{j}$ is associated with at most two bidders that win some item in OPT: bidder $o_{j_{k_{j}}}$, and at most one bidder $\left(o_{j_{i}}\right)$ that is competing on an item $j$, where $j$ is the item that $o_{j}\left(=o_{j_{i+1}}\right)$ is competing on in $A L G$. To finish the proof, we only have to show that $v_{o_{j_{j}}} \leq v_{p_{j}}$ and $v_{o_{j_{i}}} \leq v_{p_{j}}$. Since $o_{j_{k_{j}}}$ and $p_{j}$ both compete for slot $j$ (possibly they are the same bidder) and $p_{j}$ wins, $v_{o_{j_{j}}} \leq v_{p_{j}}$. Now we show the second claim. When $o_{j_{i+1}}$ arrives, $o_{j_{i}}$ is already competing on slot $j$; as $o_{j_{i+1}}$ chooses to compete on slot $j$ rather than slot $j^{\prime}$ which is also in its interval, it must be that the current candidate for slot $j$ has value at least $v_{o_{j_{i}}}$. But the eventual winner of slot $j, p_{j}$, can only have a larger value; i.e. $v_{o_{i}} \leq v_{p_{j}}$

\subsection{A Prompt Randomized Mechanism}

In this section we present a randomized prompt $O(1)$-competitive mechanism for the online scheduling problem in the value-only model. The analysis of the competitive ratio of the mechanism is related to a variant of the following balls-and-bins question:

Balls and Bins (intervals version): $n$ balls are thrown to $n$ bins, where the $i$ th ball is thrown uniformly at random to bins in the interval $W_{i}=\left[a_{i}, d_{i}\right]$. We are given that the balls can be placed in a way such that all bins are filled, and each ball $i$ is placed in exactly one bin in $\left[a_{i}, d_{i}\right]$. What is the expected number of full bins?

The theorem below proves that for every valid selection of the $a_{i}$ 's and $d_{i}$ 's in expectation at least $\frac{1}{10}$ of the bins will be full (notice that in the scheduling problem the "balls" have weights). There is a gap between this ratio and the worst example we know: in Subsection 2.3 we present an example in which at most $\frac{11}{24}$ of the bins are full in expectation. Improving the analysis of the balls and bins question will almost immediately imply an improvement in the guaranteed competitive ratio of the mechanism.

\section{The Mechanism}

1. When bidder $i$ arrives, assign it to exactly one item in $W_{i}$ to compete on uniformly at random.

2. At time $j$ conduct a second-price auction on item $j$ among all the bidders that were selected to compete on item $j$ in the first stage. 
Theorem 2.2 The mechanism is prompt and truthful, and guarantees a competitive ratio of $\frac{1}{10}$.

Proof: To see that the mechanism is truthful, recall that in the value-only model the arrival time and the departure time of each bidder are public information. It follows that the identity of bidders competing on a certain item is determined only by the outcome of the random coin flips. It is well known that a second-price auction is truthful, and thus we conclude that the mechanism is truthful. Clearly, the mechanism is prompt since the price is determined by the second-price auction which is conducted before allocating the item to the winning bidder.

We now turn to analyzing the competitive ratio of the mechanism. Instead of analyzing the competitive ratio of the mechanism, we will analyze the competitive ratio of the following process. In addition to the input of the mechanism, the input of the process consists also of "forbidden" sets $S_{1} \subseteq W_{1}, \ldots, S_{n} \subseteq W_{n}$. Later we will see how to construct these sets in a way that guarantees a constant competitive ratio.

1. For each bidder $i$ that won an item in the optimal solution, select exactly one item $j$ in $W_{i}$ to compete on uniformly at random. If $j \in S_{i}$ then bidder $i$ is not competing on any item at all.

2. At time $j$ allocate item $j$ to one bidder $i$, where bidder $i$ is selected uniformly at random from the set of all bidders that are competing on item $j$.

We will compare runs of the mechanism and the process in which the same random coins are used in Step 1. We argue that the competitive ratio of the mechanism is at least as good as the competitive ratio of the process. To see this, observe that in the first step we are restricting ourselves only to bidders that won an item in the optimal solution. Furthermore, some of these bidders are eventually not competing on any item at all. Also, the bidder that is assigned item $j$ is selected uniformly at random from the set of the bidders that are competing on item $j$, while in Step 2 of the mechanism the bidder with the highest valuation is assigned item $j$. Obviously, the mechanism does at least as well as the process. We will need the following technical lemma:

Lemma 2.3 Let $C_{j}$ be the random variable that denotes the number of bidders competing on item $j$ (the congestion of item $j$ ). Let $U_{i, j}$ be the random variable that gets the value of the utility of bidder $i$ from winning item $j$ (that is, $v_{i}$ if bidder $i$ wins item $j$, and 0 otherwise). Then,

$$
E\left[U_{i, j} \mid i \text { is competing on item } j\right] \geq \frac{v_{i}}{E\left[C_{j}\right]+1}
$$

Proof: We start by bounding from above $E\left[C_{j} \mid i\right.$ is competing on item $\left.j\right]$. That is, the expected congestion of item $j$ given that bidder $i$ is competing on item $j$. We first observe that the expected congestion produced by all other bidders apart from bidder $i$ cannot exceed $E\left[C_{j}\right]$, since the item chosen for each bidder to compete on is selected independently. Taking into account that we are given that bidder $i$ is already competing on item $j$, we conclude that $E\left[C_{j} \mid i\right.$ is competing on item $\left.j\right] \leq E\left[C_{j}\right]+1$.

Now we prove the main part of the lemma. Notice that $E\left[U_{i, j} \mid i\right.$ is competing on item $\left.j\right]=$ $\operatorname{Pr}[i$ won item $j \mid i$ is competing on item $j] \cdot v_{i}$. Let $E$ denote the set of all coin flips in which bidder $i$ is competing on item $j$ (observe that each event $e \in E$ occurs with equal probability). We let $n_{j}(e)$ denote the congestion of item $j$ in event $e$.

$$
E\left[U_{i, j} \mid i \text { is competing on item } j\right]=\Sigma_{e \in E} \frac{v_{i}}{|E| \cdot n_{j}(e)} \geq \frac{v_{i}}{E\left[C_{j}\right]+1}
$$

where the first equality is by the definition of expectation, and the second inequality is by the convexity of the function $\frac{1}{x}$, and Jensen's inequality. 
As evident from the lemma, if the expected congestion of all items that are in bidder $i$ 's time window is $O(1)$, then bidder $i$ 's expected utility is $\Theta\left(v_{i}\right)$. Unfortunately, it is quite easy to construct instances in which for every $i, S_{i}=\emptyset$ and some items face super-constant congestion. Instead, we will specify for each bidder $i$ a set of items $S_{i}$, of size at most half of the size of his time window. We will see that by a proper choice of the $S_{i}$ 's the expected congestion of every item is bounded by 4 .

Then, as each bidder $i$ (that participates in the optimal solution) has a probability of at least one half of competing on some item, by Lemma 2.3 bidder $i$ recovers in expectation at least $\frac{1}{2} \cdot \frac{1}{E\left[C_{j}\right]+1}$ of his value; by Lemma 2.4 this bidder receives in expectation at least $\frac{1}{10}$ of his value. Using the linearity of expectation, we conclude that the mechanism is $\frac{1}{10}$-competitive.

Lemma 2.4 There exist sets $S_{1}, \ldots, S_{n}$ such that for each bidder $i, S_{i} \subseteq W_{i}$, and $\left|S_{i}\right| \leq \frac{\left|W_{i}\right|}{2}$, and for each item $j, E\left[C_{j}\right] \leq 4$.

Proof: The proof of the lemma consists of $m$ stages. In each step we will consider bidders with time windows of length exactly $t$, where $t$ will take values in descending order from $m$ to 1 . We will show for each bidder $i$ with $\left|W_{i}\right|=t$ how to construct his set $S_{i}$. By the end of each step, we will be guaranteed that if $\left|W_{i}\right| \geq t$, then for each item in $W_{i} \backslash S_{i}$, the expected congestion is at most 4 .

Let us start by handling the case where $t \geq \frac{m}{2}$. Fix some bidder $i$ with $W_{i} \geq \frac{m}{2}$. Recall that we are considering only bidders that get an item in the optimal solution. The number of items is $m$, so we need to take into account at most $m$ bidders. Observe that since $W_{i} \geq \frac{m}{2}$, the average expected congestion of an item in $W_{i}$ cannot exceed 2. We let $S_{i}$ be the set of all items in $W_{i}$ for which the expected congestion is at most 4. By simple Markov arguments, $\left|S_{i}\right| \leq \frac{\left|W_{i}\right|}{2}$. We now have that for every bidder $i$ with $\left|W_{i}\right| \geq \frac{m}{2}$, and for each $j \in W_{i} \backslash S_{i}, E\left[C_{j}\right] \leq 4$.

Let us now consider Step $t$, where $t<\frac{m}{2}$. We start by considering the congestion due to bidders with time windows of length at most $t$, and then we will see that our analysis remains almost the same when including bidders with larger time windows.

Fix some bidder $i$ with $\left|W_{i}\right|=t$. We now bound from above the total congestion of the items in $W_{i}$. In the optimal solution, there are at most $t$ bidders that won an item in $W_{i}$. Their contribution to the congestion of $W_{i}$ is bounded from above by assuming that each one is competing on items in $W_{i}$ time window with probability 1 . Hence, the total contribution of these bidders is at most $t$.

Consider the bidders that won one item $j, a_{i}-t \leq j \leq a_{i}-1$, in the optimal solution. (Our analysis will only improve if $a_{i}-t \leq 0$.) Clearly, if bidder $b$ won item $j$ in the optimal solution, it must be the case that item $j$ is within $b$ 's time window. Since a bidder is selected to compete on an item uniformly, it is easy to verify that his contribution to the expected congestion of $W_{i}$ is maximized when his arrival time is $j$ and his departure time is $j+t-1$. (Recall that we are only considering bidders with time window of size at most $t$.) In this case, his contribution to the expected congestion of $W_{i}$ is $\frac{j+t-a_{i}}{t}$. Summing over all bidders (with time windows of size at most $t$ ) that won one item $j, a_{i}-t \leq j \leq a_{i}-1$, we get that the total contribution of these bidders is at most $\frac{t}{2}$.

Similarly, the total contribution of bidders with time windows of size at most $t$ that won items $d_{i}+1$ to $d_{i}+t$ in the optimal solution is at most $\frac{t}{2}$. It is easy to see that all other bidders with time windows of at most $t$ contribute nothing to the expected congestion of items in $W_{i}$. In total, we get that the total expected congestion of items in $W_{i}$ (due to bidders with time window of length at most $t$ ) is at most $\frac{t}{2}+\frac{t}{2}+t=2 t$, and thus the average expected congestion due to these items is at most 2 .

As before, we let $S_{i}$ be the set of all items in $W_{i}$ for which the expected congestion is at most 4 . Again, standard Markov arguments assure that $\left|S_{i}\right| \leq \frac{\left|W_{i}\right|}{2}$. We now have that for every bidder $i$ with $\left|W_{i}\right|=t$, and for each $j \in W_{i} \backslash S_{i}$, the average expected congestion incurred by bidders with time windows of size at most $t$ is at most 4 . We still need to take into account the congestion incurred by bidders with time windows larger than $t$. Here we observe that by our construction of the $S_{i}$ 's, these bidders can only contribute to the congestion of items with an expected congestion of at most 4 . 
Therefore, we claim that for each bidder $i$ with $\left|W_{i}\right| \geq t$, and $j \in W_{i} \backslash S_{i}$, we have that $E\left[C_{j}\right] \leq 4$. We finish the proof of the lemma by considering smaller values of $t$, down to $t=1$.

\subsection{A Bad Example}

The following example shows that the mechanism presented has a competitive ratio strictly worse than $\frac{1}{2}$. The example is an instance of the balls and bins question presented earlier. For $1 \leq i \leq \frac{n}{3}$, we let $W_{i}=\left[i, \frac{2 n}{3}\right]$. For $\frac{n}{3}<i \leq n$, we let $W_{i}=\left[\frac{n}{3}, i\right]$.

The probability that bin $i$ in $\left[1, \frac{n}{3}\right]$ will be empty is:

$$
\begin{gathered}
\operatorname{Pr}\left[\text { no ball falls to bin } i \in\left[1, \frac{n}{3}\right]\right]=\Pi_{t=1}^{i} \operatorname{Pr}\left[\text { ball } t \text { does not fall to bin } i \in\left[1, \frac{n}{3}\right]\right] \\
\qquad=\Pi_{t=1}^{i}\left(1-\frac{1}{\frac{2 n}{3}-t+1}\right)=\Pi_{t=1}^{i}\left(\frac{\frac{2 n}{3}-t}{\frac{2 n}{3}-t+1}\right)=\frac{\frac{2 n}{3}-i}{\frac{2 n}{3}}
\end{gathered}
$$

We now calculate the expected number of empty bins in the range $\left[1, \frac{n}{3}\right]$. Observe that the probability of bin $i \in\left[1, \frac{n}{3}\right]$ to be equal to the probability of bin $n-i+1$. Thus, the expected number of empty bins in $\left[1, \frac{n}{3}\right]$ is equal to the expected number of empty bins in $\left[\frac{2 n}{3}, n\right]$ :

$$
\sum_{t=1}^{\frac{n}{3}} \frac{\frac{2 n}{3}-t}{\frac{2 n}{3}}=\frac{\frac{n}{3}\left(\frac{2 n}{3}-1+\frac{n}{3}\right)}{2 \cdot \frac{2 n}{3}}=\frac{n-1}{4}
$$

Next we handle bins in the range $\left[\frac{n}{3}, \frac{2 n}{3}\right]$. Due to reasoning similar to the previous calculations, the probability that no ball $i, \frac{n}{3} \leq i \leq \frac{2 n}{3}$, falls into bin $t$ in this range is $\frac{t-\frac{n}{3}}{\frac{n}{3}}$. The probability that no ball $i, 1 \leq i \leq \frac{n}{3}$ falls to bin $t$ is $\Pi_{j=1}^{\frac{n}{3}}\left(1-\frac{1}{\frac{2 n}{3}-i+1}\right)=\frac{\frac{n}{3}}{\frac{2 n}{3}}=\frac{1}{2}$. Similarly, the probability that no ball $i$, $\frac{2 n}{3} \leq i \leq n$ falls to bin $t$ is $\frac{1}{2}$. Thus, with probability $\frac{t-\frac{n}{3}}{\frac{n}{3}} \cdot \frac{1}{4}$ no ball falls into bin $t, \frac{n}{3} \leq t \leq \frac{2 n}{3}$.

To conclude, the expected number of empty bins in the ranges $\left[1, \frac{n}{3}\right]$ and $\left[\frac{2 n}{3}, n\right]$ together is $\approx \frac{n}{2}$. The expected number of empty bins in $\left[\frac{n}{3}, \frac{2 n}{3}\right]$ is $\Sigma_{t=\frac{n}{3}}^{\frac{2 n}{3}} \frac{t-\frac{n}{3}}{\frac{n}{3}} \cdot \frac{1}{4} \approx \frac{1}{8} \cdot \frac{n}{3}$. In total, about $\frac{13}{24}$ of the bins are empty in expectation. We note that this constant can be somewhat increased by recursively applying this construction on balls in the middle third (and keeping the other balls' time windows the same). Details are omitted from this extended abstract.

\subsection{Limitations of Deterministic Tardy Mechanisms}

Here we show that the prompt mechanism of Subsection 2.1 is optimal. In order to develop some intuition about tardy mechanisms, we start by showing that the greedy mechanism of [7] is tardy.

Recall that the greedy mechanism allocates item $t$ to the bidder with the highest valuation that is present at time $t$ (and has not been assigned any item yet). Consider the following example: two bidders, red and green, arrive at time 1. The red bidder has a value of 10 for winning an item, and a departure time of time 5 . The green bidder has a value of 6 and a departure time of 1 . We will consider two scenarios: in the first one, four bidders arrive at time 2, each of them with value 100 and a departure time of 5 . In the second scenario, there are no more arrivals. First, observe that the greedy mechanism will assign the red bidder the first item. To see that the red bidder cannot learn his payment immediately, recall the following characterization of the payment in single-parameter mechanisms: the payment of a winning bidder is equal to the minimum value he can bid and still win.

In order to win an item in the first scenario, the red bidder must declare a value of at least 6 , and therefore this is his payment in this scenario. However, in the second scenario a declaration of 0 will 
make him win the second item. The mechanism cannot distinguish between the two scenarios when the red bidder wins at time 1 , and thus cannot determine the payment at time 1 . We conclude that the greedy mechanism is tardy.

The following proposition shows that every tardy deterministic mechanism for the scheduling problem achieves a competitive ratio of no better than $\frac{1}{2}$. The proof can be found in the appendix.

Proposition 2.5 Every tardy deterministic mechanism for the scheduling problem (even in the valueonly model) has a competitive ratio of no better than $\frac{1}{2}$.

\section{New Bounds for Randomized Mechanisms in the General Model}

\subsection{Limitations of Randomized Mechanisms in the Generalized Model}

In this section we prove that every randomized mechanism for the scheduling problem in the generalized model cannot guarantee a competitive ratio better than $\frac{1}{2}$. In the deterministic setting, Lavi and Nisan [9] showed that a deterministic truthful mechanism can guarantee only a trivial competitive ratio.

The proof of this theorem uses Yao's principle, which essentially says that the competitive ratio of the best randomized mechanism is equal to the competitive ratio of the best deterministic mechanism over a worst-case distribution of inputs.

Theorem 3.1 Every truthful mechanism for the scheduling problem in the generalized model cannot guarantee a competitive ratio better than $\frac{1}{2}$.

Proof: The proof begins by presenting a distribution over the input. We will see that no deterministic truthful mechanism can achieve a competitive ratio better than $\frac{2}{3}$ on this distribution. By Yao's principle, every randomized mechanism guarantees a competitive ratio no better than $\frac{2}{3}$. Later, we will extend this distribution to attain the ratio claimed in the theorem.

Before presenting the distribution itself, let us start by developing the technical tools needed for proving the bound. The next lemma shows that the decision whether to bid for an item must be made very "early". This property will play a crucial role for proving the bound. The proof of the lemma is in the appendix.

Lemma 3.2 Fix some mechanism for the online scheduling problem. Then, for every bidder that arrives at time $t$, there exists a price $p_{i}$ (that depends only on the bids of the other bidders that arrive by time $t)$ such that:

- If $p_{i}<v_{i}$ then $i$ is guaranteed to win some item in the future.

- If $p_{i}>v_{i}$, and $p_{i} \neq \infty$ then $i$ does not win any item at all.

- If $p_{i}=\infty$ then $i$ may or may not win the current item, independently of $i$ 's bid (i.e., independently of $i$ 's value or departure time).

By letting $p_{i}$ be $\infty$ the mechanism either "postpones" the decision whether to assign this bidder an item or not, or decides to assign $i$ the current item, regardless of $i$ 's bid. For example, in the randomized mechanism we present in Subsection 3.2, some bidders never get an item, no matter what their value or departure time are. We also note that although a somewhat similar lemma appears in [9], our lemma is different in several aspects: first, our lemma is weaker than [9] since we are working in a setting in which a distribution on the input is known, while [9] "alters" the input according to the algorithm. 
Second, in the randomized setting we also have to consider the case where the algorithm "postpones" the decision regarding a certain bidder ${ }^{1}$.

Let us now describe a distribution for which no deterministic algorithm can obtain a competitive ratio better than $\frac{2}{3}$. The distribution takes two parameters, $c_{1}$ and $c_{2}$, that represent values, and another parameter $t$ that determines the time during which this distribution is "active".

BasicDist $\left(t, c_{1}, c_{2}\right): m$ bidders arrive at time $t$, each one with a departure time $m$ and a value of $c_{2}$ for winning an item. The actual distribution is obtained from the basic distribution as follows; Exactly one of the following events is chosen with probability $\frac{1}{3}$ :

1. Exactly one of the bidders that arrived at time $t$ is selected uniformly at random to have a value of $c_{1}$ and a departure time of $t$.

2. $m-1$ additional bidders arrive at time $t+1$, each one with value $c_{1}$.

3. The basic distribution is left unchanged. I.e., no more arrivals, changes of values, etc.

The idea behind this distribution is that the main contribution to the welfare can come from each of the three scenarios with equal probability. However, every mechanism can handle up to two scenarios, and provides a competitive ratio of 0 in case the remaining scenario occurs. The next lemma makes this intuition formal.

Lemma 3.3 Every deterministic mechanism obtains a competitive ratio of at most $\frac{2}{3}$ on BasicDist $\left(1, c_{1}, 1\right)$, where $c_{1}>>1$.

Proof: Let $p_{1}$ be the fraction of the bidders such that given that all other bids are $c_{2}$, the bidder will be offered a price below 1 . Similarly, let $p_{2}$ denote the fraction of the bidders such that given that all other bids are $c_{2}$, the bidder will be offered a price above $c_{2}$, but below $c_{1}$.

Let us now consider the expected utility in each of the three scenarios possible in the distribution. If Scenario 1 occurs, then the competitive ratio we achieve depends on whether we postpone the "large" bidder. Postponing him (and this occurs with probability $1-p_{1}-p_{2}$ ) will result in a competitive ratio close to 0 . Otherwise, the competitive ratio will be close to 1 . Thus the expected competitive ratio in this case is $p_{1}+p_{2}$.

By Lemma $3.2^{2}$, we must assign all bidders that are offered a price below $c_{2}$ an item. Clearly, the expected contribution of these bidders is $m \cdot p_{1}$. If Scenario 2 occurs, the best we can do is to allocate the remaining $m \cdot\left(1-p_{1}\right)$ slots to the high-value bidders that arrive at time 2 . Observe that $m \cdot p_{1}$ of the high-value bidders are not assigned at all, and thus we lost a fraction of about $p_{1}$ of the total welfare. The expected competitive ratio in this case is $1-p_{1}$.

If Scenario 3 occurs, then we lose (again, by the lemma) all bidders that are offered a price larger than $c_{2}$, but are not postponed. As before, the expected number of these is $1-p_{2}$, and this is the expected competitive ratio we get for this scenario.

Recall that each scenario occurs with probability of exactly $\frac{1}{3}$. In total, the expected competitive ratio we get is at most:

$$
\frac{1}{3} \cdot\left(p_{1}+p_{2}\right)+\frac{1}{3} \cdot\left(1-p_{1}\right)+\frac{1}{3} \cdot\left(1-p_{2}\right)=\frac{2}{3}
$$

\footnotetext{
${ }^{1}$ Notice that this is not a problem in the deterministic setting, since if there exist a scenario in which the mechanism postpones a newly arrived bidder to a later stage, we can always set the value of this bidder to $\infty$ and his departure time to the current item, and then the mechanism cannot guarantee any competitive ratio at all.

${ }^{2}$ Lemma 3.2 requires that the prices will be different than the value of the bidder. For this to be true we might randomly add some "noise" to all values of $c_{2}$. By choosing high enough precision, the chance of a price to be equal to a value is negligible. We omit this from the description of the distribution (and the analysis) to keep the presentation simple.
} 
Let us now specify a recursive family of distributions that will be used in the proof of this theorem. We assume that $r$ is some large enough constant and positive integer. The parameter that determines a specific member of the family of distributions is a positive integer $t$.

MainDist $t_{t}\left(c_{0}, c_{1}, \ldots, c_{r}\right): m-(r-t)$ bidders arrive at time $r-t+1$, each one with departure time $m$ and a value of $c_{r-t+1}$ for winning an item. Exactly one of the following events will now occur:

1. With probability $\frac{1}{2 t+1}$, exactly one of the bidders that arrived at time $r-t+1$ is selected uniformly at random to have a value of $c_{r-t}$ and a departure time of $r-t+1$.

2. With probability $\frac{1}{2 t+1}$, MainDist is left unchanged. I.e., no more arrivals, changes of values, etc.

3. With probability $1-\frac{2}{2 t+1}$, the next stages are determined by MainDist $_{t+1}\left(c_{0}, c_{1}, \ldots, c_{r}\right)$.

We also need to define MainDist :

MainDist ${ }_{0}\left(c_{0}, c_{1}, \ldots, c_{r}\right): m-r$ bidders are arriving at time $r$, each one with departure time $m$ and a value of $c_{0}$ for winning an item.

Lemma 3.4 Fix some positive integer $r$. Every deterministic truthful mechanism cannot obtain an expected competitive ratio better than $\frac{r+1}{2 r+1}$ on MainDist $t_{r}\left(c_{0}, \ldots, c_{r}\right)$, where $c_{r}>>\ldots>>c_{0}$.

The proof of this lemma is in the appendix.

\subsection{A Randomized Mechanism for the Generalized Model}

Designing mechanisms in the generalized model is much more complicated, as the main tools we have for designing mechanisms in multi-parameter settings, VCG, cannot be applied in an online setting. To overcome this obstacle, we extend the mechanism from Section 2. Before describing the mechanism itself, we note again that a mechanism that provides a competitive ratio that is equal to the logarithm of the highest valuation, can be easily constructed. However, this requires us to know in advance the highest valuation. In addition, if this value is exponentially high, the guaranteed competitive ratio might be polynomially large. The mechanism below overcomes both issues.

Suppose that $m$ is known in advance. Then, a poly-logarithmic randomized mechanism can be constructed as follows: For each bidder $i$ we select uniformly at random a value $d_{i}^{\prime}$ that will take an integer value between 1 and $\log m$. We will assume that the length of the time window of the $i$ th bidder is $2^{d_{i}^{\prime}}$ and run the mechanism from Section 2. From now on, we will only consider bidders for which we "guessed" the length of their time window correctly. That is, bidders in which $2^{d_{i}^{\prime}-1} \leq\left|W_{i}\right| \leq 2^{d^{\prime}}$. The expected fraction of these bidders is $\Theta\left(\frac{1}{\log m}\right)$, and clearly these bidders also hold in expectation a logarithmic fraction of the welfare achieved in the optimal solution. For these bidders, the analysis of the mechanism yields, with minor deviations, that we are able to recover a constant fraction of the value they hold. In total, we get a mechanism that guarantees a competitive ratio of $\Theta\left(\frac{1}{\log m}\right)$. The following mechanism handles the case where $m$ is not known in advance, and improves the competitive ratio guarantee. A formal description and a proof can be found in Appendix A.4.

\section{References}

[1] Yair Bartal, Francis Y. L. Chin, Marek Chrobak, Stanley P. Y. Fung, Wojciech Jawor, Ron Lavi, Jiří Sgall, and Tomáš Tichý. Online competitive algorithms for maximizing weighted throughput of unit jobs. In STACS, pages 187-198, 2004. 
[2] Francis Y. L. Chin and Stanley P. Y. Fung. Online scheduling with partial job values: Does timesharing or randomization help? Algorithmica, 37(3):149-164, 2003.

[3] Marek Chrobak, Wojciech Jawor, Jiří Sgall, and Tomáš Tichý. Improved online algorithms for buffer management in QoS switches. In ESA, pages 204-215, 2004.

[4] Matthias Englert and Matthias Westermann. Considering suppressed packets improves buffer management. In In SODA'O\%.

[5] Eric J. Friedman and David C. Parkes. Pricing wifi at starbucks: issues in online mechanism design. In EC '03: Proceedings of the 4th ACM conference on Electronic commerce, pages 240-241, New York, NY, USA, 2003. ACM Press.

[6] Andrew V. Goldberg, Jason D. Hartline, Anna R. Karlin, Mike Saks, and Andrew Wright. Competitive auctions. Games and Economic Behavior, 2006.

[7] Mohammad T. Hajiaghayi, Robert Kleinberg, Mohammad Mahdian, and David C. Parkes. Adaptive limited-supply online auctions. In Proc. ACM Conf. on Electronic Commerce, pages 165-174, 2005.

[8] A. Kesselman, Zvi Lotker, Yishay Mansour, Boaz Patt-Shamir, Baruch Schieber, and Maxim Sviridenko. Buffer overflow management in QoS switches. In STOC, pages 520-529, 2001.

[9] Ron Lavi and Noam Nisan. Online ascending auctions for gradually expiring items. In SODA'05.

[10] Ron Lavi and Noam Nisan. Competitive analysis of incentive compatible on-line auctions. In $A C M$ Conference on Electronic Commerce, pages 233-241, 2000.

[11] Fei Li, Jay Sethuraman, and Clifford Stein. Better online buffer management. In In SODA'0\%.

[12] Mohammad Mahdian and Amin Saberi. Multi-unit auctions with unknown supply. In EC '06: Proceedings of the 7th ACM conference on Electronic commerce, pages 243-249, New York, NY, USA, 2006. ACM Press.

[13] Ahuva Mu'alem and Noam Nisan. Truthful approximation mechanisms for restricted combinatorial auctions. In $A A A I-02,2002$.

[14] Noam Nisan and Amir Ronen. Algorithmic mechanism design. In STOC, 1999.

\section{A Appendix}

\section{A.1 Proof of Proposition 2.5}

Proof: Consider the following setting: two bidders arrive at time 1, each of them with a value of 1 , and a departure time of time 2. Suppose there are no more arrivals of other bidders. Any mechanism that achieves a competitive ratio better than 2 must assign one bidder the first item, and the other item to the second bidder. Let $a$ be bidder that was assigned the first item, and $b$ be the bidder that was assigned the second item.

Claim A.1 Let $M$ be a tardy mechanism with a finite competitive ratio. In the scenario described above, there is no declaration of a value $v_{b}$ that makes bidder $b$ win the first item. 
Proof: Denote the payment of bidder $b$ for winning the second item with a declaration of 1 by $p_{b}$. Observe that $p_{b}<1^{3}$. We consider two cases, one in which $b$ declares a value of $w>1$, and one in which $b$ declares a value of $w<1$.

Suppose that bidder $b$ raises his bid from 1 to $w$, and consequently is assigned the first item. The mechanism is not tardy, so the payment of bidder $b$ is determined immediately. Suppose, for a contradiction, that this payment is higher than $p_{b}$. In this case, if bidder $b$ 's true value is $w$, he can improve his profit by declaring a value of 1 , and be assigned the second item. Hence the payment must be at most $p_{b}$. Clearly, the payment can not be strictly less than $p_{b}$, since otherwise if $b$ 's true value is 1 , he has an incentive to declare a value of $w$ and increase his profit. Thus the payment must be equal to $p_{b}$, but now we will see that this cannot be the case. Consider the following setting: $b$ 's true value is 1 , and therefore he does not win the first item. On time 2 a bidder $c$ that is going to depart at time 2 and value $w^{\prime}>>w$ arrives. In order to maintain a finite competitive ratio the mechanism must assign bidder $c$ the second item. We get that if bidder $b$ 's true value is 1 , he has an incentive to declare a value of $w$ (and therefore win the first item for a payment of $p_{b}$ ), and the mechanism is not truthful.

Let us now handle the other case, where $b$ bids a value $w, w<1$, and win the first item (notice that his payment is less than 1). As before, if a bidder $c$ with a departure time of 2 , and a very high value arrives at time 2, then the mechanism must assign $c$ the second item in order to guarantee a finite competitive ratio. If bidder $b$ 's true value is 1 , he is an incentive to declare $w$ instead, and win the first item. The claim follows.

Let us now alter the scenario described above, and let $b$ 's value be $w>>1$. By the claim, the mechanism will not assign bidder $b$ the first item. Consider the case in which at time 2 bidder $c$ with a departure time of 2 and a value of $w$ arrives. The total welfare the mechanism achieves is at most $1+w$, while the optimal welfare is $2 \cdot w$. The proposition follows.

\section{A.2 Proof of Lemma 3.2}

Proof: We start by fixing a bidder $i$ that arrives at time $t$. First, assume this bidder has a departure time of $t$. In this setting the private information of bidder $i$ consists only of one number: $v_{i}$. By well-known arguments for single parameter settings, there exists a threshold value that depends only on the bids of the other bidders such that if $i$ bids above it he wins an item, and otherwise he gets no item. Let $p_{i}$ to be this threshold value. The lemma trivially follows for this case.

Now consider the case in which the departure time of $i$ is not necessarily $t$. If $v_{i}>p_{i}$ and bidder $i$ does not win any item at all, then bidder $i$ has an incentive to declare a departure time of $t$, contradicting the truthfulness of the mechanism. Thus, if $v_{i}>p_{i}$ the mechanism must guarantee bidder $i$ that he will win some item before his departure time.

Consider now the case where $v_{i}<p_{i}$. Suppose that bidder $i$ wins some item with price of $p^{\prime}$. Clearly, $p^{\prime}<p_{i}$, otherwise bidder $i$ 's profit is negative. Now assume that instead of bidder $i$ we have another bidder $i^{\prime}$ with the same arrival time and departure time as bidder $i$ but with value $v_{i^{\prime}}$, $v_{i}<p_{i}<v_{i^{\prime}}$. Observe that $i^{\prime}$ has an incentive to declare a value of $v_{i}$ (instead of $v_{i^{\prime}}$ ). Again, we obtained a contradiction to the truthfulness of the mechanism.

The third condition holds trivially by the properties of truthful mechanisms.

\section{A.3 Proof of Lemma 3.4}

Proof: We will see that for any positive integer $t$ every deterministic mechanism can not obtain a competitive ratio better than $\frac{r+1}{2 r+1}$ on $\operatorname{MainDist}_{r}\left(c_{0}, \ldots, c_{r}\right)$. We will do so by induction on $t$. First ob-

\footnotetext{
${ }^{3}$ Indeed, $p_{b}$ can also be equal to 1 . In this case we can add some "noise" to the value of the bidders described above, and get a strict inequality.
} 
serve that if $t=1$ then the our claim is correct because MainDist ${ }_{1}\left(c_{0}, \ldots, c_{r}\right)$ is identical to BasicDist, and the competitive ratio follows. We now prove the lemma for $t>1$.

Let $p_{1}$ denote the fraction of the bidders such that given that all other bids are $c_{r-t+1}$, the bidder is offered a price below $c_{r-t+1}$. Let $p_{2}$ denote the fraction of the bidders such that given that all other bids are $c_{r-t+1}$ the bidder is offered a price above $c_{r-t+1}$, but still below $c_{r-t+1}$. Let $p_{1}=\frac{\Sigma_{i} p_{1}^{i}}{n^{\prime}}$, and $p_{2}=\frac{\Sigma_{i} p_{2}^{i}}{n^{\prime}}$, where $n^{\prime}$ denotes the number of bidder that arrived at time $t$.

Let us now analyze the expected competitive ratio under any possible scenario. If Scenario 1 occurs, then with probability of $p_{1}+p_{2}$ we do not postpone the high-value bidder. (If we do, we attain no reasonable competitive ratio.) In this case the expected competitive ratio is $p_{1}+p_{2}$.

In Scenario 2, we lose all bidders that are offered a value which was too high. The fraction of welfare that the the rest of the bidders hold is at most $1-p_{2}$, and this is an upper bound for the expected competitive ratio of this stage.

Finally, if Scenario 3 occurs, then the welfare is completely dominated by bidders that arrive according to MainDist $t_{t+1}$. We lose a fraction of $\left(1-p_{1}\right)$ of the bidders that we are committing to serve. By induction, the expected competitive ratio we can achieve now is bounded from above by $\frac{t}{2 t-1}\left(1-p_{1}\right)$.

To finish the proof, we calculate the overall expected competitive ratio for the current value of $t$ :

$$
\frac{1}{2 t+1} \cdot\left(p_{1}+p_{2}\right)+\frac{1}{2 t+1} \cdot\left(1-p_{2}\right)+\frac{2 t-1}{2 t+1} \cdot\left(\frac{t}{2 t-1}\left(1-p_{1}\right)\right)=\frac{t+1}{2 t+1}-\frac{\left(p_{1} \cdot(1-t)\right.}{2 t+1} \leq \frac{t+1}{2 t+1}
$$

\section{A.4 A Randomized for the Generalized Model}

\section{The Mechanism:}

1. Add each bidder that arrives to one of the following groups: to the statistics group $S T A T$ with probability $\frac{1}{2}$, to the competitors group $C$ with probability $\frac{1}{4}$, or to the impatient bidders group $I$ with probability $\frac{1}{4}$.

2. Let $m^{\prime}$ be the largest departure time among bidders that are currently in STAT. (That is, $m^{\prime}=\max _{i \in S T A T} d_{i}$.) For each bidder in $C$ with arrival time of $t$ select a departure time uniformly at random from the following set: $t, t+1, t+3, t+7, \ldots, t+2^{4 \log m^{\prime}}-1$.

3. For each bidder $i$ in $C$ with arrival time of $t$ select uniformly at random an item in $\left[t, t+d_{i}^{\prime}\right]$ to compete on.

4. Conduct a second-price auction on item $t$ among all bidders that were selected to compete on item $t$, and all bidders in $I$ that arrived in time $t$. Allocate item $t$ to the winner.

Theorem A.2 There exists a prompt truthful $\Theta\left(\frac{1}{\log m}\right)$-competitive mechanism.

Proof: The truthfulness of the mechanism trivially follows because each bidder participates in at most one second price auction whose participants are determined only by the outcome of the random coins. Also note that bidders in STAT are never assigned any items, and thus have no incentive to misreport their preferences. The promptness of the mechanism is also obvious. All that is left is to show is that the mechanism is $\Theta\left(\frac{1}{\log m}\right)$ competitive.

The analysis is divided into two different cases. In the first case, there is a bidder $i$ that holds at least a fraction of $\frac{1}{16 \log m}$ of the optimal welfare. In this case, with probability $\frac{1}{4}$ we will select bidder $i$ to the group $I$. (If this is not the case, we assume that the value of the solution obtained is 0 .) Look at time $t$ in which bidder $i$ arrives. We conduct a second-price auction on item $t$, with the participation of 
bidder $i$. Surely, the winner in this auction holds a value of at least a fraction of $\frac{1}{16 \log m}$ of the optimal welfare. To conclude this case, with probability $\frac{1}{2}$ we are able to recover at least $\frac{1}{16 \log m}$ fraction of the welfare, thus the mechanism is indeed $\Theta\left(\frac{1}{\log m}\right)$ competitive in this case.

Let us now consider the case where no bidder holds a fraction of $\frac{1}{16 \log m}$ of the optimal welfare. We first claim that since no bidder dominates the optimal welfare, with probability $1-o(1)$ the bidders in $C$ hold a fraction of at least $\frac{1}{8}$ of the optimal welfare. The claim follows from the fact that each bidder has a probability of $\frac{1}{4}$ to be selected to $C$ (proof of claim omitted - see, e.g., [6]). We will now see that we can recover at least a logarithmic fraction of the welfare of bidders in $C$.

Look at the bidders that won some item in the optimal solution, and order them by their arrival time, $o_{1}, \ldots, o_{m}$. Now select at most $\log m$ bidders $b_{1}, \ldots, b_{\log m}$ in the following way: $o_{1}$ is set to be $b_{1}$. We set $b_{2}$ to be $o_{t}$, where $t$ is the smallest index such that $d_{o_{t}}>2 d_{o_{1}}$, and so on. Notice that indeed we select at most $\log m$ bidders this way. Observe that for any $t$, if $b_{t}$ is selected to STAT (and this happens with probability of $\frac{1}{2}$ ), we will guess correctly the departure time $d_{i}^{\prime}$ (up to a constant factor) with probability of at least $\Theta\left(\frac{1}{\log m}\right)$, for all bidders $i$ such that $b_{t} \leq i \leq b_{t+1}$. In other words, for each bidder $i$ in $C \backslash \cup_{t=1}^{\log m}\left\{b_{t}\right\}$ we guess his departure time correctly with probability of $\Theta\left(\frac{1}{\log m}\right)$.

All that is left to prove is that the set $C \backslash \cup_{t=1}^{\log m}\left\{b_{t}\right\}$ holds a constant fraction of the optimal welfare. If we prove this, we will be able to use the analysis of the mechanism of Section 2 to show that we recover a constant fraction of the welfare obtained by bidders in $C \backslash \cup_{t=1}^{\log m}\left\{b_{t}\right\}$ whose departure times were guessed correctly. This will be enough to show that the mechanism is $\Theta\left(\frac{1}{\log m}\right)$ competitive.

With probability of $1-o(1)$, the value of bidders in $C$ is at least $\frac{1}{8}$ of the value of the optimal solution. The value of bidders in $\cup_{t=1}^{\log m}\left\{b_{t}\right\}$ is at most $\log m \cdot \frac{O P T}{16 \log m}$. Thus the value that bidders in $C \backslash \cup_{t=1}^{\log m}\left\{b_{t}\right\}$ hold is at least $\frac{1}{16}$ of the optimal solution. This concludes the proof of the theorem. 